\title{
ESTUDO DA DIFUSÃO E FRAGILIZAÇÃO POR HIDROGÊNIO EM AÇO INOXIDÁVEL SUPERDUPLEX*
}

\author{
Ana Maria Pérez Ceballos ${ }^{1}$ \\ Marcella Lage ${ }^{2}$ \\ Kioshy Santos de Assis ${ }^{3}$ \\ Jorge Andrés Calderón Gutiérrez 4 \\ Oscar Rosa Mattos ${ }^{5}$
}

\section{Resumo}

Os aços inoxidáveis duplex e superduplex são caracterizados por uma estrutura bifásica contendo ilhas de austenita precipitadas numa matriz de ferrita. Estes aços possuem boas propriedades mecânicas e boa resistência à corrosão. No entanto, estes aços podem apresentar fragilização por hidrogênio. No desenvolvimento deste trabalho se estudou a difusão e fragilização por hidrogênio de um aço inoxidável superduplex SAF 2507 nos estados como recebido e tratado térmicamente. Para avaliar a influência da microestrutura sobre a difusão do hidrogênio foram realizados ensaios de permeação electroquímica de hidrogênio. Observou-se que o coeficiente de difusão aparente aumenta em função da temperatura de recozimento. A influência de hidrogênio na resistência à fratura do material recozido a $1300^{\circ} \mathrm{C}$ foi estudada a partir de ensaios de tenacidade à fratura (CTOD). Determinou-se que a resistência à fratura do material diminui consideravelmente quando o mesmo é submetido a uma carga de hidrogênio. A presença de hidrogênio também modificou o mecanismo de fratura do material, observou-se uma fratura completamente dúctil no material que foi testado em ausência de hidrogênio e uma fratura frágil, caracterizada por clivagem, foi observada no material hidrogenado. Determinou-se que a fase austenítica pode ser fragilizada sob certas condições de carga de hidrogênio.

Palavras-chave: Aços superduplex; Difusão de hidrogênio; Fragilização por hidrogênio; CTOD.

\section{STUDY OF HYDROGEN DIFUSSION AND EMBRITTLEMENT IN SUPER DUPLEX STAINLESS STEEL}

\section{Abstract}

Duplex stainless steels are characterized by a biphasic structure containing austenite islands precipitated in the ferrite matrix. These steels have good mechanical properties and good corrosion resistance. However, these materials are susceptible to hydrogen embrittlement. In this work has been studied the hydrogen diffusion and embrittlement of super duplex stainless steel SAF 2507 in the material as received and annealed. To evaluate the influence of microstructure on the diffusion have been performed hydrogen electrochemical permeation tests. It was observed that the apparent diffusion coefficient increases with annealing temperature. The influence of hydrogen on toughness of the annealed material was studied using CTOD testing. It was determined that the fracture toughness of the material decreases considerably when it is subjected to hydrogen charge. The presence of hydrogen also changed the material fracture mechanism, a completely ductile fracture of the material has been observed in the material tested in the absence of hydrogen and a brittle fracture, characterized by cleavage, was observed in the hydrogenated material. It has been determined that the austenitic phase could be embrittle by hydrogen under certain conditions.

Keywords: Superduplex stainless steel; Hydrogen diffusion; Hydrogen embrittlement; CTOD.

1 Candidata a Doutora em Engenharia, Mestre, Eng. Metalúrgica, Grupo CIDEMAT, Depto. de Eng. de Materiais, Faculdade de Engenharia, Universidad de Antioquia, Medellín, Antioquia, Colômbia.

2 Estudante de Mestrado, Engenheira de Materiais, Laboratório LNDC, DMM-PEMM/COPPE/UFRJ, Rio de Janeiro, RJ, Brasil.

3 Candidato a Doutor, Mestre, Engenheiro Metalúrgico, Laboratório LNDC, DMM-PEMM/COPPE/UFRJ, Rio de Janeiro, RJ, Brasil.

4 Doutor em Ciências, Engenheiro Metalúrgico, Professor, Grupo CIDEMAT, Departamento de Engenharia de Materiais, Faculdade de Engenharia, Universidad de Antioquia, Medellín, Antioquia, Colômbia.

5 Doutor em Ciências, Engenheiro Metalúrgico, Professor, Laboratório LNDC, DMM-PEMM/COPPE/UFRJ, Rio de Janeiro, RJ, Brasil. 


\section{INTRODUÇÃO}

Os aços inoxidáveis duplex (AID) podem ser definidos como uma família de aços caracterizada por uma microestrutura constituída por duas fases: austenita (CFC) e ferrita (CCC). Na prática, o termo refere-se a ligas duplex em que tanto a ferrita quanto a austenita estão presentes em volumes relativamente grandes e em frações aproximadamente iguais (não menos do que $30 \%$ de cada) [1, 2]. As proporções exatas de ferrita e austenita são definidas através do controle da composição química e do tratamento térmico [1,3]. Os AID têm vantagens comparativas em relação aos aços inoxidáveis, entre as quais se incluem: maior resistência mecânica, maior resistência à corrosão e menor preço, principalmente devido ao menor teor de níquel na liga [2, 4]. Em comparação com os aços inoxidáveis ferríticos, os AID têm resistência à corrosão semelhante e apresentam maior tenacidade [4]. Em geral, os aços que tem a ferrita como fase predominante tem excelente resistência à corrosão, devido à elevada solubilidade do $\mathrm{Cr}$ nessa fase. Essas características explicam o aumento da utilização destes aços em várias aplicações industriais tais como a indústria de petróleo e a indústria química. A fim de aumentar a resistência à corrosão localizada foram desenvolvidos, há alguns anos, os aços inoxidáveis superduplex (AISD). A principal diferença entre os graus duplex e superduplex refere-se aos maiores teores de $\mathrm{Cr}, \mathrm{Ni}$, Mo e $\mathrm{N}$ encontrados nestes últimos. Além disso, os AISD contem pequenas quantidades de outros elementos, normalmente não encontrada em graus duplex tais como $\mathrm{Cu}$ e W [5]. Este tipo de aço foi desenvolvido especialmente para aplicações que envolvem água do mar, indústrias químicas e petroquímicas em que são necessárias alta resistência mecânica e alta resistência à corrosão em ambientes extremamente agressivos, tais como ambientes ácidos contendo cloretos. Apesar do bom desempenho contra a corrosão de AID e AISD em ambientes altamente corrosivos, estes podem falhar devido à fragilização por hidrogênio, dependendo da concentração de hidrogênio presente, das condições mecânicas e de serviço e do tratamento térmico da liga. Os átomos de hidrogênio podem ser gerados por corrosão localizada, por carga catódica associada com processos de proteção catódica ou por interação galvânica, por exemplo, em contacto com um aço de carbono [6]. A susceptibilidade das ligas metálicas à degradação por hidrogênio depende, principalmente, da absorção de átomos de hidrogênio na fase metálica, da mobilidade destes átomos e da distribuição do hidrogênio entre a rede cristalina e os defeitos estruturais os quais agem como armadilhas; os dois últimos fatores estão diretamente relacionados com as características microestruturais do material [7]. A interação de hidrogênio com AID e AISD tem sido caracterizada usando a técnica de permeação eletroquímica de hidrogênio [1, 6, 8, 9]. Foi determinado que a difusão de hidrogênio nestes aços ocorre principalmente através da fase ferrítica porque o coeficiente de difusão do hidrogênio nesta fase é muito maior (até cinco ordens de magnitude) do que o encontrado na austenita. Além disso, a solubilidade do hidrogênio na austenita é 2500 vezes maior do que na ferrita, de modo que, se espera que a difusão do hidrogênio seja através da ferrita e que o elemento fique preso na austenita. A difusão do hidrogênio nos AID e AISD é muito menor em relação à difusão nos aços inoxidáveis ferríticos, isto é devido, principalmente, ao aumento da distância a ser percorrida gerado pela presença dos grãos de austenita os quais dificultam o caminho, assim como a possibilidade do hidrogênio ficar preso tanto e na fase austenítica quanto nos limites de grão. A forma, tamanho e espaçamento interaustenítico pode modificar tanto a difusão quanto o aprisionamento de hidrogênio. 
Em geral, ilhas finas e dispersas de austenita dificultam a difusão e favorecem o aprisionamento de hidrogênio [10]. Vários estudos têm sido realizados para avaliar a fragilização por hidrogênio dos AID e AISD, em geral, esses estudos avaliaram a resposta do material à aplicação de uma carga em ensaios mecânicos como Slow Strain Rate Testing (SSRT) com hidrogênio pré-carregado tanto em forma gasosa quanto catódica $[1,11]$. A tenacidade à fratura deste tipo de aços tem sido estudada por testes como Crack Mouth Opening Displacement (CMOD) [12]. Em todos os casos observou-se uma perda de ductilidade dos aços duplex e superduplex, quando foram carregados com hidrogênio, também foi observado que a fratura ocorre por um mecanismo de clivagem o quase clivagem associado à fratura frágil.

\section{MATERIAIS E MÉTODOS}

O material estudado corresponde a um aço inoxidável superduplex SAF 2507. A influência da microestrutura sob a difusão de hidrogênio foi avaliada a partir de ensaios de permeação electroquímica de hidrogênio em amostras do material como recebido tanto na secção longitudinal (CRL) quanto na transversal (CRT), e recozido a 1050 (TT1050) e $1300^{\circ} \mathrm{C}$ (TT1300) durante 1 hora sob atmosfera de argônio controlada. A microestrutura do material foi caracterizada pela técnica metalográfica usando microscópio óptico e microscópio eletrônico de varredura (MEV). A fim de revelar a microestrutura, as amostras foram imersas numa solução de Behara modificada $\left(60 \mathrm{ml}\right.$ de $\mathrm{H}_{2} \mathrm{O}, 40 \mathrm{ml}$ de $\mathrm{HCl}, 1 \mathrm{~g}$ de $\mathrm{K}_{2} \mathrm{~S}_{2} \mathrm{O}_{5}$ ) durante 60 segundos. A quantificação das fases (ferrita e austenita), o espaçamento interaustenítico e à contagem das ilhas de austenita foram determinadas usando 20 micrografias obtidas a 200X. O espaçamento interaustenítico foi determinado de acordo com o procedimento estabelecido pelas normas ASTM E112-96 e DVN-RP-F112. Para os ensaios de permeação eletroquímica de hidrogênio se usaram amostras polidas de $0,4 \mathrm{~mm}$ de espessura. O lado de detecção de hidrogênio foi revestido com uma película de níquel de 20 um. Os testes de permeação eletroquímica foram realizados utilizando uma célula do tipo Devanathan e Stachurski [13] constituída pelo material estudado como eletrodo de trabalho, um fio de platina como contra eletrodo e um eletrodo de calomelano saturado (ECS) como eletrodo de referência. Na célula de geração se utilizou uma solução de $\mathrm{NaOH} 0,1 \mathrm{M}+2 \mathrm{mgL}^{-1} \mathrm{As}_{2} \mathrm{O}_{3}$; a densidade de corrente catódica utilizada para gerar hidrogênio foi de $10 \mathrm{mAcm}^{-2}$. O lado de detecção foi mantido sob controle potenciostático, foi aplicado um potencial constante de oxidação (+300mV vs ECS), não só antes do início da permeação (a fim de permitir que a corrente de passivação diminuía para um valor quase estacionário), mas também durante a permeação de hidrogênio (a fim de oxidar o hidrogênio emergente). Durante todo o ensaio foi injetado nitrogênio gasoso em ambas as células. A temperatura foi mantida constante a $25^{\circ} \mathrm{C}$. A partir das curvas experimentais de permeação foi calculado o coeficiente de difusão aparente de hidrogênio (Dapp) usando o método do tempo tangente (breakthrough time) [14]. Os ensaios de tenacidade à fratura (CTOD) foram realizados de acordo as Normas BS 7448-1:1991 Fracture mechanics toughness tests, Part 1: Method for determination of KIc, critical CTOD and critical $J$ values of metallic materials e ASTM E1820-01 Standard Test Method for Measurement of Fracture Toughness [15, 16]. As dimensões das amostras foram selecionadas com o objetivo de ter uma área de carregamento de hidrogênio semelhante à utilizada nos ensaios de permeação eletroquímica. Três amostras foram submetidas a teste de flexão em três pontos para o material recozido a $1300^{\circ} \mathrm{C}$ com e sem carga de hidrogênio. O tempo de 
hidrogenação foi de 2 meses aproximadamente. Os ensaios de CTOD foram realizados utilizando uma máquina servo hidráulica da marca Instron modelo 8801 com uma célula de carga de capacidade máxima de $100 \mathrm{kN}$. O teste de fratura foi realizado na mesma máquina em que foi feito o prétrincamento, sob controle de deslocamento a uma velocidade do travessão de $1 \mathrm{~mm} \mathrm{~min}^{-1}$ para o material que não foi submetido à carga de hidrogênio e de $0,01 \mathrm{~mm} \mathrm{~min}^{-1}\left(0,0067 \mathrm{MPa} \mathrm{m}^{1 / 2} \mathrm{~s}^{-1}\right)$ para as amostras hidrogenadas. Esta velocidade de ensaio é aproximadamente 100 vezes menor do que o valor mínimo especificado pela norma BS 7448-1 (0,5 MPa $\mathrm{m}^{1 / 2} \mathrm{~s}^{-1}$ ) e foi selecionada com o intuito de permitir a interação entre o material e o hidrogênio preso durante a deformação causada pelo ensaio de CTOD.

\section{RESULTADOS E DISCUSSÃO}

\subsection{Caracterização do material}

Em todos os casos, a microestrutura está constituída por ilhas de austenita dispersas em uma matriz semicontinua de ferrita. A fase escura corresponde à matriz ferrítica. A microestrutura do material no estado CRL é caracterizada por ilhas alongadas de austenita (fase clara). O material no estado CRT e TT1050 apresenta uma microestrutura semelhante, onde as ilhas de austenita são mais arredondadas em comparação com o observado na secção longitudinal. O tratamento térmico a alta temperatura (TT1300) produz um aumento da fase ferrítica e a austenita perdeu completamente a estrutura de grãos alongados. Não foi observada a precipitação de outras fases após o tratamento térmico. A figura 1 apresenta a microestrutura obtida para cada estado metalúrgico estudado.
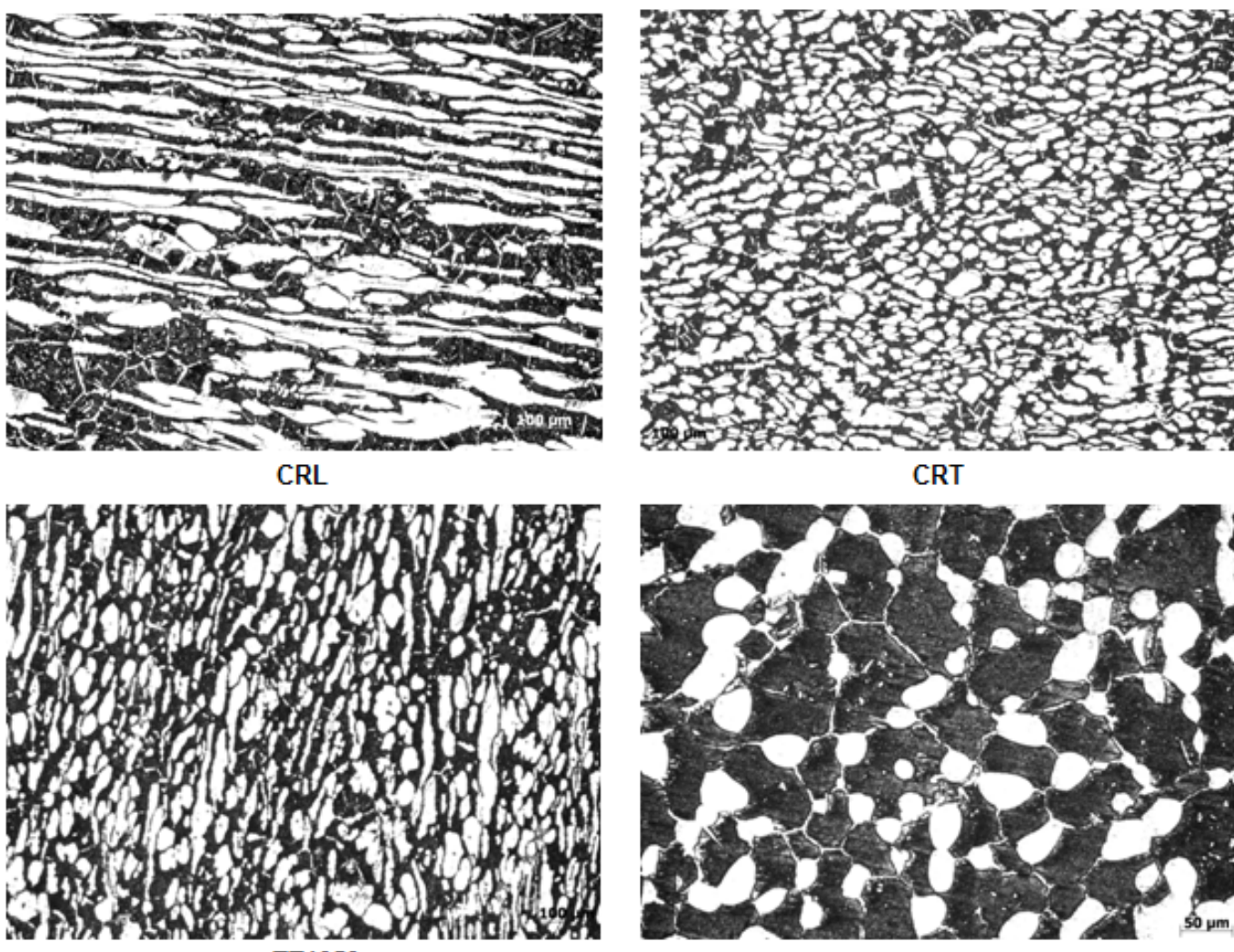

CRT

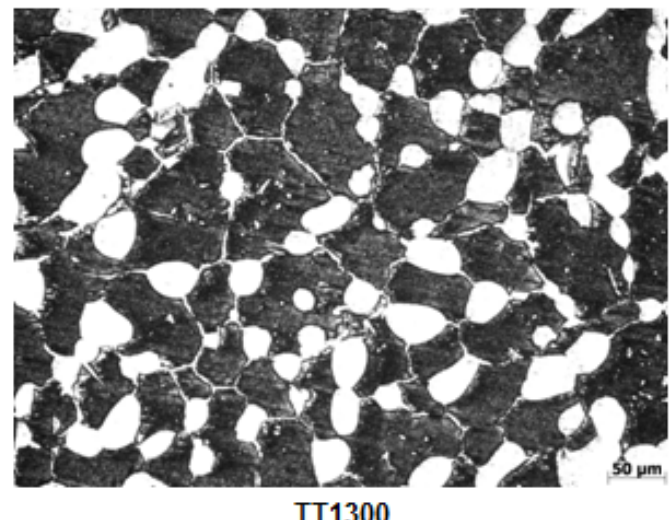

Figura 1. Microestrutura do material SAF2507 para cada estado metalúrgico estudado. 


\subsection{Quantificação das fases}

A partir das micrografias foi calculada a fração volumétrica de austenita presente em todas as condições metalúrgicas estudadas. Os resultados são mostrados na tabela 1.

\begin{tabular}{ccc}
\multicolumn{2}{c}{ Tabela 1. Fração volumétrica das fases } \\
\hline $\begin{array}{c}\text { Estado } \\
\text { Metalúrgico }\end{array}$ & $\begin{array}{c}\text { Fração } \\
\text { volumétrica de } \\
\text { austenita (\%) }\end{array}$ & $\begin{array}{c}\text { Fração } \\
\text { volumétrica de } \\
\text { ferrita (\%) }\end{array}$ \\
\hline CRL & $54,76 \pm 5,19$ & $45,24 \pm 5,19$ \\
\hline CRT & $52,78 \pm 3,58$ & $47,22 \pm 3,58$ \\
\hline TT1050 & $42,60 \pm 4,05$ & $57,40 \pm 4,05$ \\
\hline TT1300 & $26,37 \pm 2,62$ & $73,63 \pm 2,62$ \\
\hline
\end{tabular}

A fração volumétrica de austenita foi reduzida com o tratamento térmico. O aumento da temperatura de recozido aumenta a quantidade de ferrita. A fração volumétrica das fases presentes está em concordância com a literatura; para o SDSS SAF 2507 na condição como recebido têm sido relatadas percentagens de austenita entre $45 \mathrm{e}$ $49 \%[1,17]$. Para um DSS Urano $B 50 ®$ recozido a $1050^{\circ} \mathrm{C}$ durante uma hora foi obtida uma percentagem de austenita de 33\% [6] enquanto que em um DSS H25N5M foi encontrada uma percentagem de austenita de $40 \%$ após um tratamento de recozimento a $1050^{\circ} \mathrm{C}$ durante 90 minutos [8]. As diferenças encontradas durante este estudo podem ser consequência de variações na composição química dos aços mencionados.

\subsection{Determinação do espaçamento interaustenítico}

O espaçamento interaustenítico está relacionado com a distância entre as ilhas de austenita e é determinado pelo tamanho de grão de ferrita. A Tabela 2 mostra os resultados obtidos.

Tabela 2. Espaçamento interaustenítico

\begin{tabular}{ccc}
\hline $\begin{array}{c}\text { Estado } \\
\text { Metalúrgico }\end{array}$ & $\begin{array}{c}\text { Espaçamento } \\
\text { interaustenítico } \\
{[\boldsymbol{\mu} \boldsymbol{\mu m}]}\end{array}$ & $\begin{array}{c}\text { Acurácia relativa } \\
(\% \mathbf{H})\end{array}$ \\
\hline CRL & $11,761 \pm 0,59$ & 5,2 \\
\hline CRT & $12,670 \pm 0,93$ & 5,7 \\
\hline TT1050 & $14,213 \pm 0,83$ & 5,4 \\
\hline TT1300 & $47,554 \pm 5,47$ & 8,2 \\
\hline
\end{tabular}

O espaçamento interaustenítico é maior no material que foi tratado termicamente do que no material como recebido. É notável o elevado aumento do espaçamento interaustenítico no material recozido a uma temperatura elevada; o resultado está de acordo com as modificações da microestrutura observadas nessa condição metalúrgica (TT 1300).

\subsection{Permeação eletroquímica de hidrogênio}

As curvas de permeação obtidas para o AISD SAF 2507 são apresentadas na figura 2. 


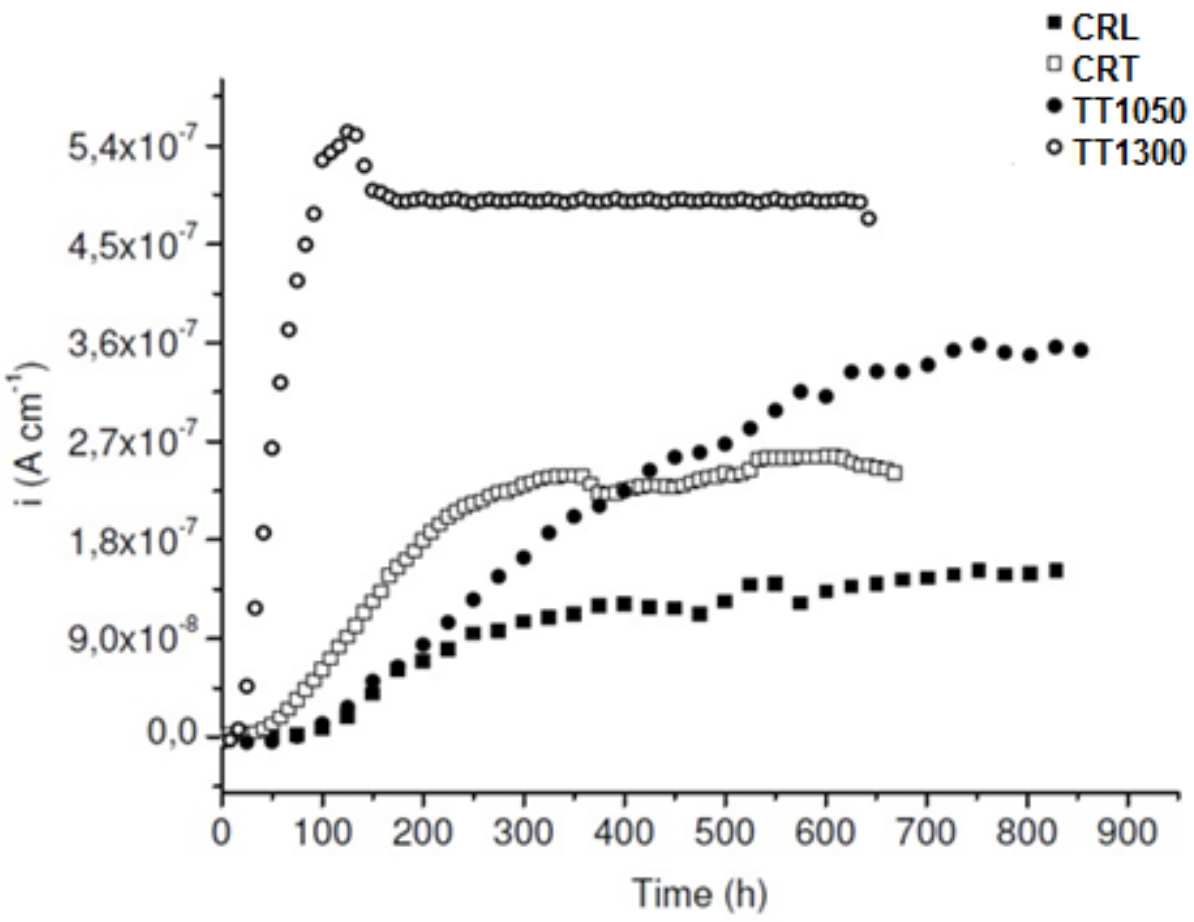

Figura 2. Curvas de permeação para o material SAF 2507 em diversas condições metalúrgicas.

A partir das curvas de permeação foi determinada a corrente de permeação no estado estacionário, o tempo tangente ( $\left.\mathrm{t}_{\mathrm{b}}\right)$ e o coeficiente de difusão aparente para cada estado metalúrgico estudado. Na Tabela 3 se apresentam os resultados obtidos.

Tabela 3. Parâmetros calculados a partir das curvas de permeação eletroquímica

\begin{tabular}{|c|c|c|c|c|}
\hline $\begin{array}{c}\text { Estado } \\
\text { Metalúrgico }\end{array}$ & $\begin{array}{l}\text { Corrente de permeação } \\
\text { no estado estacionário } \\
\text { [A] }\end{array}$ & $\begin{array}{l}\text { Tempo tangente } \\
\left(\mathrm{t}_{\mathrm{b}}\right)[\mathrm{h}]\end{array}$ & $\begin{array}{l}\text { Dapp (th) } \\
{\left[\mathrm{m}^{2} \mathrm{~s}^{-1}\right]}\end{array}$ & Desvio padrão \\
\hline $\mathrm{CRL}$ & $1,48 \mathrm{E}-6 \pm 1,35 \mathrm{E}-7$ & 142,2 & $1,44 \mathrm{E}-14$ & \multirow{4}{*}{$1,96 \mathrm{E}-15$} \\
\hline CRT & $2,47 \mathrm{E}-6 \pm 1,14 \mathrm{E}-7$ & 122,5 & $1,91 \mathrm{E}-14$ & \\
\hline TT1050 & $3,18 \mathrm{E}-6 \pm 8,42 \mathrm{E}-8$ & 131,6 & $2,16 \mathrm{E}-14$ & \\
\hline TT1300 & $5,13 \mathrm{E}-6 \pm 1,31 \mathrm{E}-8$ & 36,0 & $6,10 \mathrm{E}-14$ & \\
\hline
\end{tabular}

Um aumento na corrente de permeação no estado estacionário foi observado nas amostras recozidas. O tempo tangente é superior no material no estado como recebido; o aumento da temperatura de recozimento diminui o valor do tempo tangente. O coeficiente de difusão aparente está diretamente relacionado com a microestrutura do material. O material recozido a uma temperatura elevada (TT1300) tem um coeficiente de difusão aparente quatro vezes maior do que o material no estado como recebido na secção longitudinal e três vezes maior do que o material como recebido na secção transversal e TT1050. Além disso, verificou-se que o coeficiente de difusão aparente para o material como recebido na secção transversal é maior do que o observado no material como recebido na secção longitudinal; este fato já havia sido descrito por Hutchings et al. [6]. O valor encontrado para o material no estado CRL de 1,44E-14 $\mathrm{m}^{2} \mathrm{~s}^{-1}$ está em concordância com o valor encontrado por Alsarraf de 1,42E-14 $\mathrm{m}^{2} \mathrm{~s}^{-1}$ [1]. Turnbull obteve um coeficiente de difusão de hidrogênio aparente de $1,5 \mathrm{E}-14 \mathrm{~m}^{2} \mathrm{~s}^{-1}$ para o aço Urano $\mathrm{B} 50 \AA$ na condição como recebido a $22^{\circ} \mathrm{C}$ [6]. O AISD SAF2507 tem um maior teor de elementos de liga (incluindo $\mathrm{N}$ ) se comparado com o aço Urano $\mathrm{B} 50 \AA$, isto poderia explicar a 
diminuição do coeficiente de difusão observada no AISD SAF 2507, pois tem sido determinado que os elementos de liga em solução sólida podem se comportar como sítios de aprisionamento de hidrogênio $[18,19]$. O aumento do coeficiente de difusão de hidrogênio observado no material como recebido na secção transversal poderia ser explicado em função do espaçamento interaustenítico que para esta condição é $7 \%$ maior do que na secção longitudinal. Tem sido estabelecido que enquanto mais perto se encontrem os sítios de aprisionamento (neste caso os bordes de grão) menos hidrogênio vai entrar na matriz e os fenômenos de difusão serão mais lentos [20]. Para AISD SAF2507 recozido a 1050 e $1300^{\circ} \mathrm{C}$, não foi possível encontrar dados do coeficiente de difusão de hidrogênio na literatura.

\subsection{Tenacidade à fratura CTOD}

Na figura 3 se apresentam os gráficos obtidos durante os ensaios de CTOD para o material no estado TT1300 ao ar e hidrogenado, respectivamente. A curva obtida para o material testado ao ar tem uma forma suave e contínua, característica de materiais que exibem comportamento de fratura dúctil. Da mesma forma, pode-se ver que a curva correspondente ao material hidrogenado tem um aspecto menos suave que pode ser associado com o crescimento instável da trinca (pop-in) característico de materiais em que a fissura é iniciada de forma frágil, mas apenas se propaga uma corta distância, pois a propagação é interrompida pela presença de uma zona de maior ductilidade [21].
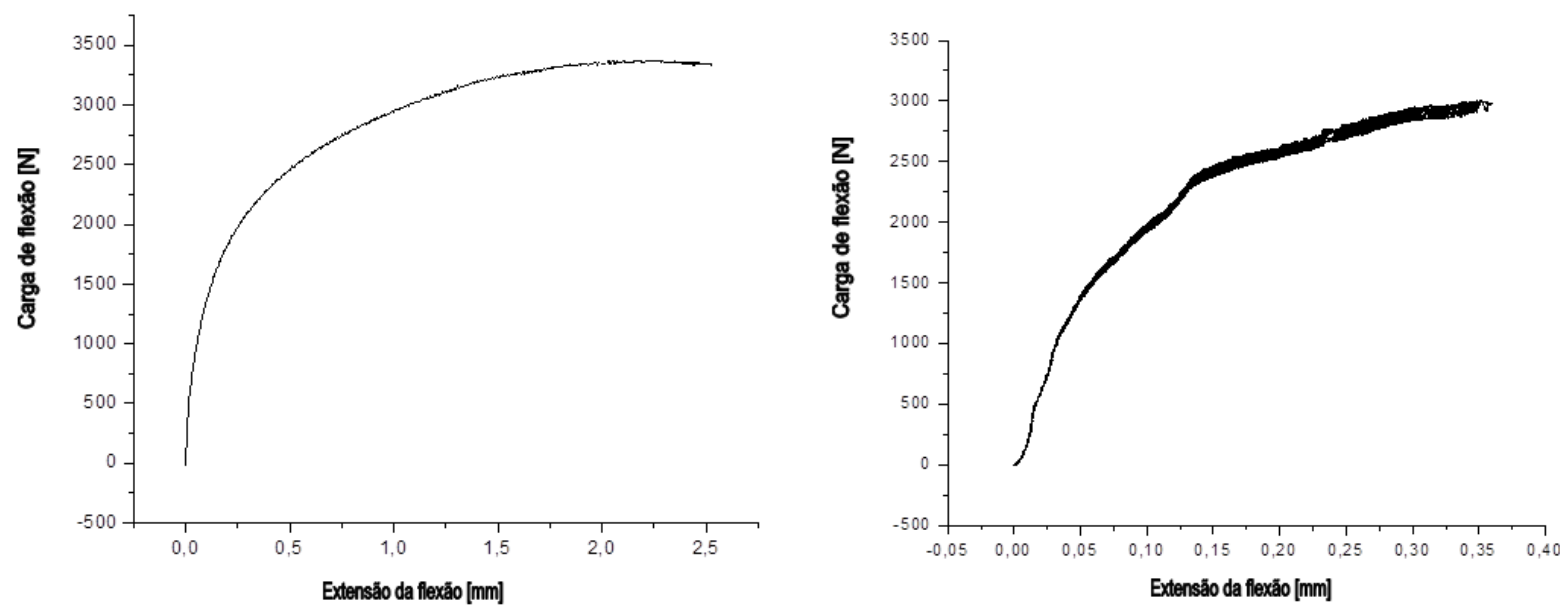

Figura 3. Carga vs Extensão da flexão para o aço SAF 2507 TT1300. A curva da esquerda corresponde ao material testado sem carga previa de hidrogênio. A curva da direita corresponde ao material hidrogenado.

Comparando os gráficos da figura 3 pode-se estabelecer que para uma carga máxima de aproximadamente $3 \mathrm{kN}$, a extensão da flexão diminui drasticamente quando o material é sujeito a carga de hidrogênio antes do ensaio de CTOD; passando de um valor de $2,5 \mathrm{~mm}$ para o material ensaiado sem hidrogênio a $0,4 \mathrm{~mm}$ no material hidrogenado. Os valores de CTOD encontrados para o material no estado TT1300 foram $\delta=0,509 \pm 0,197$ milímetros quando o material foi submetido ao ensaio sem pré-carregamento de hidrogênio e $\delta u=0,119 \pm 0,0348$ milímetros quando o material ensaiado foi previamente hidrogenado. Como esperado, o hidrogênio tem uma influência direta sobre o valor de CTOD. Neste estudo, para o material SAF 2507 no estado TT1300 se observou uma diminuição do CTOD de 
aproximadamente $77 \%$ nos corpos de prova que foram carregados com hidrogênio antes do teste, o que revela uma perda da resistência à fratura de material. A partir da análise da morfologia da superfície da fratura foi estabelecido que as amostras carregadas com hidrogênio apresentaram uma fratura frágil caracterizada pela presença de planos de clivagem, enquanto que o material não hidrogenado apresentou uma morfologia característica da fratura dúctil, como se pode observar na figura 4. Isto é, os resultados mostram uma clara fragilização do material estudado quando submetido a uma carga de hidrogênio, pois tem sido determinado que a fratura dos AISD é completamente dúctil, na ausência deste elemento; o qual foi confirmado pela forma contínua e lisa da curva obtida durante os ensaios de CTOD realizados em amostras não hidrogenados [12, 21].
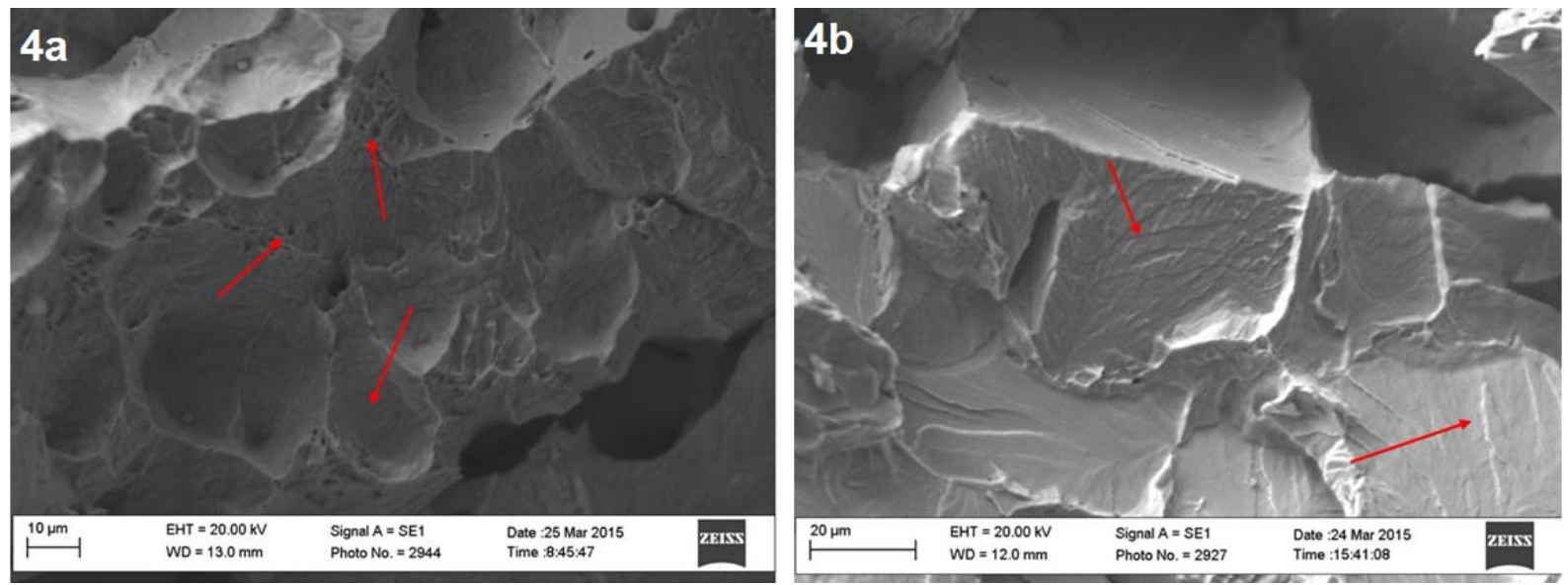

Figura 4. Superfície de fratura do aço SAF 2507 TT1300 após ensaios de CTOD. A figura 4a corresponde ao material testado sem carga previa de hidrogênio, as setas vermelhas indicam as características da fratura dúctil. A figura $4 \mathrm{~b}$ corresponde ao material hidrogenado, as setas vermelhas indicam os planos de clivagem próprios da fratura fragil.

Prasad et al. [22] encontraram uma diminuição de $11 \%$ no valor do CTOD em um aço SAF 2205 depois de ser submetido a carga catódica de hidrogênio por 8 dias. Esses autores encontraram uma morfologia mista na superfície de fratura; onde a fase austenítica possui características típicas da fratura dúctil; e a fase ferrítica tem um comportamento frágil determinado por clivagem ou quase clivagem. Oltra et ai. determinaram experimentalmente que a propagação de fissuras causadas por hidrogênio em AID é caracterizada por clivagem no plano da ferrita com uma mudança de direção e microfissuras em zigue-zague ao longo da direção <111> quando entra na fase austenítica [23]. No presente estudo, não se apresentaram alterações na superfície de fratura em relação às fases. A superfície de fratura tanto para a ferrita quanto para a austenita é caracterizada por planos de clivagem relacionados com fratura frágil. Esta observação é importante porque mostra que o hidrogênio não só difunde na ferrita, mas também pode causar fragilização da fase austenítica e é consistente com o encontrado por Olden et al., quem observou a fragilização da fase austenítica em um AID com teor de $\mathrm{Cr} 25 \%$ submetido a um potencial de proteção catódica de $-1050 \mathrm{mV}$ vs ECS numa solução de água do mar sintética recirculada a $4^{\circ} \mathrm{C}$, evidenciada pela presença de clivagem na superfície de fratura da referida fase [12]. 


\section{CONCLUSÃO}

A microestrutura tem uma influência direta sob a difusão do hidrogênio no aço inoxidável superduplex SAF 2507. Diminuindo a fração volumétrica de austenita se obtém um aumento no coeficiente de difusão aparente. Do mesmo modo, se estabeleceu que o hidrogênio difunde mais facilmente quando o fluxo é paralelo à orientação das ilhas de austenita. A tenacidade à fratura do material recozido a $1300^{\circ} \mathrm{C}$ foi drasticamente reduzida quando o material foi submetido a uma carga de hidrogênio. O mecanismo de fratura do material foi modificado pela presença de hidrogênio. No material testado sem hidrogênio foi observada uma fratura completamente dúctil. Além disso, o material que foi sujeito à carga hidrogênio apresentou planos de clivagem característicos da fratura frágil. O tempo de hidrogenação parece ser um fator determinante no mecanismo de fratura da fase austenítica. Este estudo mostrou que dita fase fratura frágil após longos períodos de hidrogenação.

\section{REFERÊNCIAS}

1. Alsarraf J. Hydrogen embrittlement susceptibility of super duplex stainless steels [Tese de doutorado]. Cranfield, Bedford: Cranfield University; 2010.

2. Nilsson J.O. Super duplex stainless steels. Materials Science and Technology, 1992. 8(8): 685-700.

3. Gun, R.N. Duplex stainless steel: Microstructure, properties and applications. Cambridge: R.N. Gunn; 1997.

4. Villalobos D, Albiter A, Maldonado C. Microstructural changes in SAF 2507 superduplex stainless steel produced by thermal cycle. Matéria (Rio J.) Online, 2009. 14: 1061-1069.

5. Comer A.J. Corrosion fatigue of a superduplex stainless steel weldment, [Tese de doutorado]. Dublin: Dublin City University; 2003.

6. Hutchings R.B, Turnbull A, May A.T. Measurement of hydrogen transport in a duplex stainless steel. Scripta Metallurgica et Materialia, 1991. 25(12): p. 2657-2662.

7. Zakroczymski T, Owczarek E. Electrochemical investigation of hydrogen absorption in a duplex stainless steel. Acta Materialia, 2002. 50(10): p. 2701-2713.

8. Owczarek E, Zakroczymski T. Hydrogen transport in a duplex stainless steel. Acta Materialia, 2000. 48(12): p. 3059-3070.

9. Turnbull A, Hutchings R.B. Analysis of hydrogen atom transport in a two-phase alloy. Materials Science and Engineering: A, 1994. 177(1-2): p. 161-171.

10. Turnbull A, Lembach-Beylegaard E, Hutchings R. Hydrogen transport in SAF 2205 and SAF 2507 duplex stainless steel. Hydrogen Transport and Cracking in Metals. The Institute of Materials, Cambridge, UK, 1995: p. 268-279.

11. Zheng W, Hardie D. The effect of hydrogen on the fracture of a commercial duplex stainless steel. Corrosion Science, 1991. 32(1): p. 23-36.

12. Olden V, Thaulowa C, Johnsena R, Østbyb E, Berstadb T. Influence of hydrogen from cathodic protection on the fracture susceptibility of $25 \% \mathrm{Cr}$ duplex stainless steel Constant load SENT testing and FE-modelling using hydrogen influenced cohesive zone elements. Engineering Fracture Mechanics, 2009. 76(7): p. 827-844.

13. Devanathan M.A, Stachurski Z. The adsorption and diffusion of electrolytic hydrogen in palladium. Proc. Roy. Soc. A,1962. 270: p. 90.

14. Boes $\mathrm{N}$, Züchner $\mathrm{H}$. Electrochemical methods for studying diffusion, permeation and solubility of hydrogen in metals. Journal of less common metals, 1976. 49: p. 223-240.

15. B.S, BS 7448-1: Fracture mechanics toughness tests - Part 1: Method for determination of Klc, critical CTOD and critical J values of metallic materials. 1991: p. 48.

16. ASTM, ASTM E1820 -13: Standard Test Method for Measurement of Fracture Toughness. 2013: p. 46. 
17. Topolska S, Labanowski J. Effect of microstructure on impact toughness of duplex and superduplex stainless steels. Journal of AMME, 2009. 36(2): p. 142-149.

18. Hagi $\mathrm{H}$, Effect of Substitutional Alloying Elements (Al, Si, V, Cr, Mn, Co, Ni, Mo) on the Diffusion Coefficient of Hydrogen in a-Iron. Mater. Trans, JIM, 1992: p. 472 - 479.

19. Pressouyre G.M, Bernstein I.M. A quantitative analysis of hydrogen trapping. Metallurgical Transactions A, 1978(11): p. 1571-1580.

20. Pressouyre G.M. A classification of hydrogen traps in steel. Metallurgical Transactions A, 1979. 10(10): p. 1571-1573.

21. Zhu X.K, Joyce J.A. Review of fracture toughness (G, K, J, CTOD, CTOA) testing and standardization. Engineering Fracture Mechanics, 2012. 85(0): p. 1-46.

22. Prasad R.C, Roychowdhury S, Kain V. Effect of hydrogen pickup on fracture behaviour of stainless steels. International Conference on Fracture. Otawa. 2009.

23. Oltra $\mathrm{R}$, Bouillot $\mathrm{C}$. Experimental investigation of the role of hydrogen in stress corrosion cracking of duplex stainless steels. Hydrogen Transport and Cracking in Metals, 1994: p. 17-26. 